\title{
Systematic Literature Review and Critical Appraisal of Health Economic Models Used in Cost-Effectiveness Analyses in Non-Alcoholic Steatohepatitis: Potential for Improvements
}

\author{
Pierre Johansen $^{1}$ (D) Daniel Howard ${ }^{2} \cdot$ Ryan Bishop $^{3} \cdot$ Søren Ilsøe Moreno ${ }^{1} \cdot$ Kristine Buchholtz $^{1}$
}

Published online: 10 January 2020

(c) The Author(s) 2020

\begin{abstract}
Background Non-alcoholic steatohepatitis (NASH) is a severe, typically progressive form of non-alcoholic fatty liver disease (NAFLD). The global prevalence of NASH is increasing, driven partly by the global increase in obesity and type 2 diabetes mellitus (T2DM), such that NASH is now a leading cause of cirrhosis. There is currently an unmet clinical need for efficacious and cost-effective treatments for NASH; no pharmacologic agents have an approved indication for NASH.

Objective Our objective was to summarise and critically appraise published health economic models of NASH, to evaluate their quality and suitability for use in the assessment of novel treatments for NASH, and to identify knowledge gaps, challenges and opportunities for future modelling.

Methods A systematic literature review was performed using the MEDLINE, Embase, Cochrane Library and EconLit databases to identify published health economic analyses in patients with NAFLD or NASH. Supplementary hand searches of grey literature were also performed. Articles published up to November 2019 were included in the review. Quality assessment of identified studies was also performed.

Results A total of 19 articles comprising 16 unique models including either NAFLD as a whole or NASH alone were included in the review. Structurally, most models had a state-transition component; in terms of health states, two different approaches to early disease states were used, modelling either progression through fibrosis stages or NAFLD/NASH-specific health states. Conditions that frequently co-exist with NASH, such as obesity, T2DM and cardiovascular disease were not captured in models identified here. Late-stage complications such as cirrhosis, decompensated cirrhosis and hepatocellular carcinoma were consistently included, but input data (e.g. costs, utilities and transition probabilities) for late-stage complications were frequently sourced from other liver disease areas. The quality of included studies was heterogenous, and only a small proportion of studies reported internal and external validation processes.

Conclusion The health economic models identified in this review are associated with limitations primarily driven by a lack of NASH-specific data. Identified models also largely overlooked the intricate association between NASH and other conditions, including obesity and T2DM, and did not capture the increased risk of cardiovascular events associated with NASH. High-quality, transparent, validated health economic models of NASH will be required to evaluate the cost effectiveness of treatments currently in development, particularly compounds that may target other non-hepatic outcomes.
\end{abstract}

\section{Introduction}

Non-alcoholic fatty liver disease (NAFLD) is an overarching term and defined as the presence of $\geq 5 \%$ hepatic steatosis in the absence of secondary causes of hepatic fat accumulation and includes both non-alcoholic fatty liver (NAFL) and

Electronic supplementary material The online version of this article (https://doi.org/10.1007/s40273-019-00881-7) contains supplementary material, which is available to authorized users.

Extended author information available on the last page of the article non-alcoholic steatohepatitis (NASH) [1]. NAFL is defined as " $\geq 5 \%$ hepatic steatosis, but without evidence of hepatocellular injury in the form of hepatocyte ballooning" [1]. A subset of patients with NAFLD have NASH, which is progressive and defined as "the presence of $\geq 5 \%$ hepatic steatosis and inflammation with hepatocyte injury, with or without fibrosis" [1]. On a global level, the prevalence of NAFLD is estimated at around $20-30 \%$ in adults [2, 3], and the prevalence of NASH is estimated at 2-5\% [2].

NASH occurs as a consequence of a state of lipid surplus in which the rate of uptake or synthesis of lipids into 


\section{Key Points for Decision Makers}

There is a paucity of data specific to non-alcoholic fatty liver disease (NAFLD)/non-alcoholic steatohepatitis (NASH) relating to cost inputs, quality of life and disease progression to inform the development of health economic models. Many currently available models rely on input data from other liver disease areas.

Identified models typically do not include cardiovascular outcomes or account for the fact that NASH often coexists alongside other conditions, including obesity and type 2 diabetes mellitus (T2DM).

The treatment benefits of pharmacologic agents currently in development for NASH may extend beyond the hepatocyte. As such, there is an unmet need for models that take cardiovascular outcomes and comorbid conditions such as obesity and T2DM into account.

Model developers are restricted by the data that are available; future studies linking surrogate outcomes to hard clinical endpoints may assist model developers in terms of modelling long-term outcomes based on available short-term data.

Identified health economic models of NASH are largely product specific; there is an unmet need for non-productspecific models, which would also facilitate comparison of findings across different analyses.

The reporting quality of identified models was heterogenous; increased transparency is needed in the development of future models to enhance credibility and acceptance with payers, policy makers and other key stakeholders.

hepatocytes exceeds the rate of export and degradation [4]. The development and rate of progression of NASH is highly heterogeneous between patients, reflecting the complex interplay between different underlying etiologic factors, including lifestyle, diet, genetics and epigenetics [5]. However, in NAFLD, several factors, including diabetes and elevated alanine aminotransferase (ALT) levels have been shown to be significant independent predictors of progression to moderate or severe fibrosis [6]. NAFLD and aspects of the metabolic syndrome share common etiologic components [7], and NAFLD is increasingly considered to represent a hepatic component of the metabolic syndrome [8].

NAFL is largely asymptomatic, and NASH may also be asymptomatic in many patients, or patients may present with non-specific symptoms such as fatigue. Patients with
NAFLD with simple steatosis may have no progression or even regression of disease, and these patients do not have an elevated mortality risk [9]. However, approximately 30-40\% of patients who develop NASH will develop fibrosis to a varying degree, and $15-20 \%$ of those with fibrosis will go on to develop cirrhosis, which is in turn associated with an elevated risk for decompensated cirrhosis, hepatocellular carcinoma (HCC), requirement for liver transplantation and liver-related mortality [10].

As NASH is a progressive disease, its increasing prevalence has notable implications in terms of the clinical and economic burden of disease on national and global levels. In the USA alone, total annual direct medical costs related to the treatment and management of patients with NAFLD (including patients with NAFL, NASH and later complication states, including fibrosis, cirrhosis and $\mathrm{HCC}$ ) are estimated at over \$US100 billion ( $€ 91$ billion; October 2019 exchange rate) (\$US1613 [€1472] per patient), whereas the corresponding figure in Germany, France and Italy combined is estimated at $€ 28$ billion ( $€ 354-1163$ per patient) [11]. Total direct costs for patients with NASH (including those with late-stage complications, i.e. fibrosis, cirrhosis and HCC) were \$US17 billion ( $€ 15.5$ billion) in the USA and $€ 5$ billion for Germany, France and Italy combined [11]. Despite the considerable clinical and economic burden of $\mathrm{NASH}$, no drugs are indicated for the treatment of NASH in either the USA or Europe, although the off-label use of pioglitazone and vitamin $\mathrm{E}$ is mentioned in both US and European guidelines [1, 12]. Several different pathways can lead to disease progression. As such, phase II/III trials of a number of drugs with different mechanisms of action are ongoing [13]. Given the rapid rise in the prevalence of NASH, an unmet clinical need exists for drugs that can arrest or reverse the progression of disease, particularly in patients progressing to fibrosis and potentially cirrhosis. Further, novel drugs with mechanisms of action that may extend beyond the hepatocyte, such as anti-inflammatory agents, insulin sensitizers or glucagon-like peptide 1 receptor agonists may have systemic effects that independently influence non-hepatic outcomes. Alongside the demonstration of clinical efficacy, in ever more cost-constrained environments, it is becoming increasingly important for novel drugs to also demonstrate long-term cost effectiveness. Such evaluations will ideally require high-quality, transparent health economic models as well as high-quality clinical, economic and quality-of-life (QoL) input data. To this end, the aim of the current review was to summarise and critically appraise published health economic models of NASH (and NAFLD) and to evaluate their quality and suitability for use in the assessment of novel treatments for NASH, as well as to identify knowledge gaps, challenges and opportunities for future modelling. 


\section{Methods}

A systematic literature review was performed using the MEDLINE, Embase, Cochrane Library and EconLit databases. The review was conducted in line with Cochrane guidelines [14], and search strategies were constructed using both medical subject headings and free-text terms (full details of the search strategies used are provided in Tables 1-7 in the Electronic Supplementary Material [ESM]). Initial searches were performed in July 2018 and updated in November 2019 to capture the most recently published articles. Supplementary hand searches of grey literature were also performed. For inclusion, studies were required to be full-text publications (data were not extracted from analyses published as abstracts alone) of health economic analyses in patients with NAFLD or NASH and published in English. No restrictions were applied in terms of interventions, comparators or outcomes. First-round screening of titles and abstracts was followed by second-round full-text screening of short-listed articles and data extraction of articles meeting the inclusion criteria. Both first- and second-round screening was performed by two independent reviewers, and disagreements relating to the inclusion or exclusion of articles were resolved by discussion and consensus or, where necessary, through consultation with a senior reviewer.

Data extraction was performed using pre-designed data extraction tables in Microsoft Word. Data extracted from each article included model characteristics, health state utility values, costs, treatment effects, model validation and uncertainty (stochastic and parameter level). Strengths, limitations and key findings of included analyses were also evaluated. The quality of included analyses was assessed using the Philips et al. [15] checklist, which is a 53-item checklist assessing model structure and data quality.

\section{Results}

\subsection{Literature Search Results}

The combined original (July 2018) and updated (November 2019) literature searches across the four included databases identified a total of 539 hits, of which 97 were duplicates, therefore yielding a total of 442 unique hits (Fig. 1). A further 369 hits were excluded during the first-round screening of titles and abstracts, and 56 were excluded during the second-round full-text screening. A further two articles were identified through supplementary searches of grey literature. As such, a total of 19 articles [11, 16-33] (capturing 16 different models) were included in the review; summary details of identified studies are shown in Table 1.

A total of 13 articles assessed health economic outcomes associated with different screening or diagnostic strategies [16, 18-21, 26-33]; five assessed outcomes with different treatments, including lifestyle intervention, pioglitazone, vitamin $\mathrm{E}$ and obeticholic acid as well as bariatric surgery for overweight/obese patients with NASH [17, 22-25]. The remaining article examined the overall clinical and economic burden of disease and as such was a non-comparative study [11].

\subsection{Model Approach and Structure}

Analyses identified in the review examined either NAFLD as a whole, NASH alone or both NAFLD and NASH as

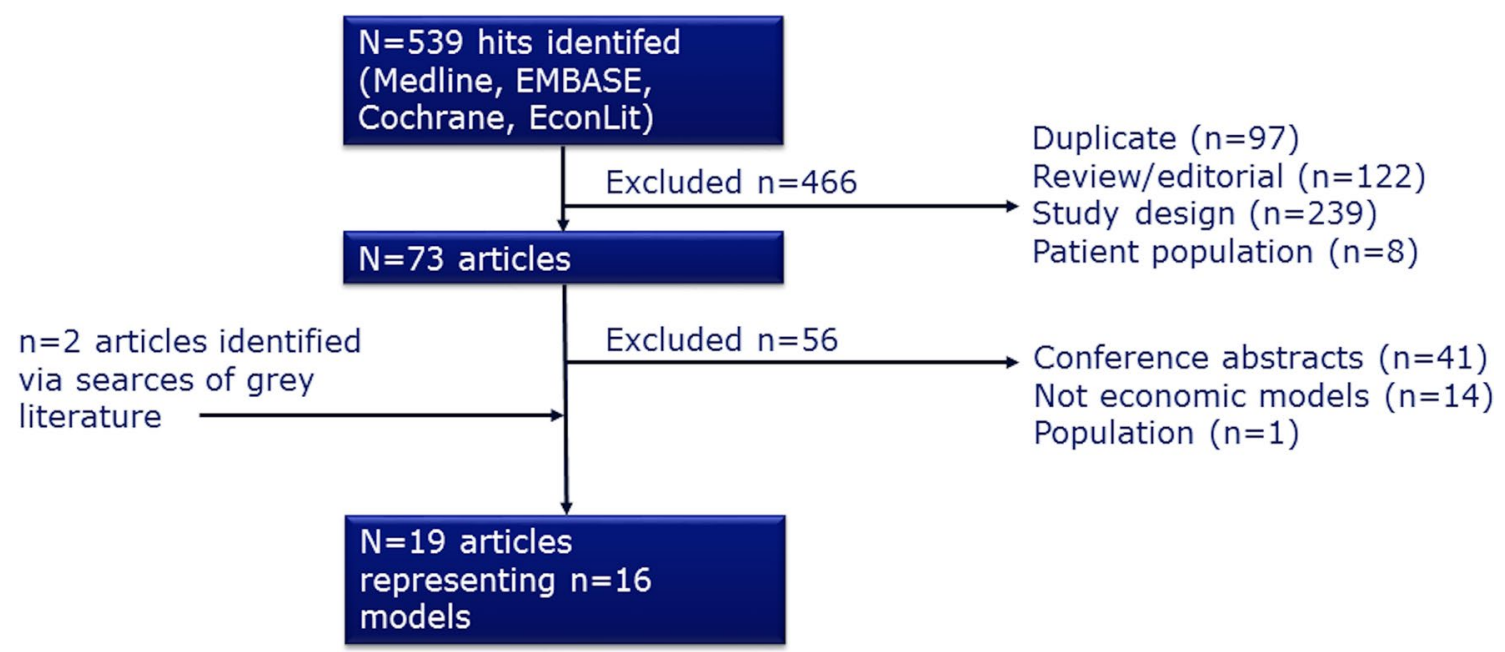

Fig. 1 Overview of literature search process 


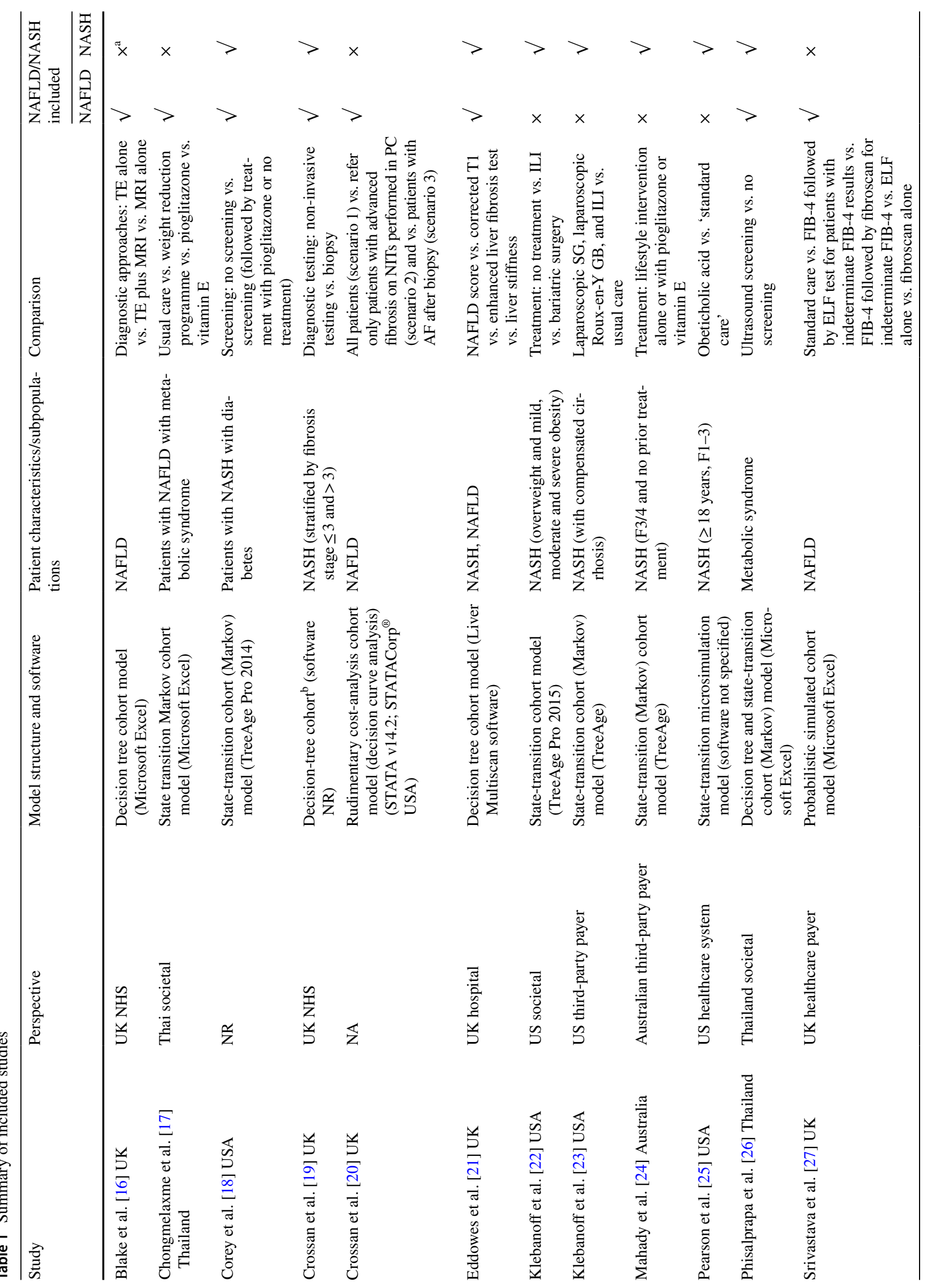




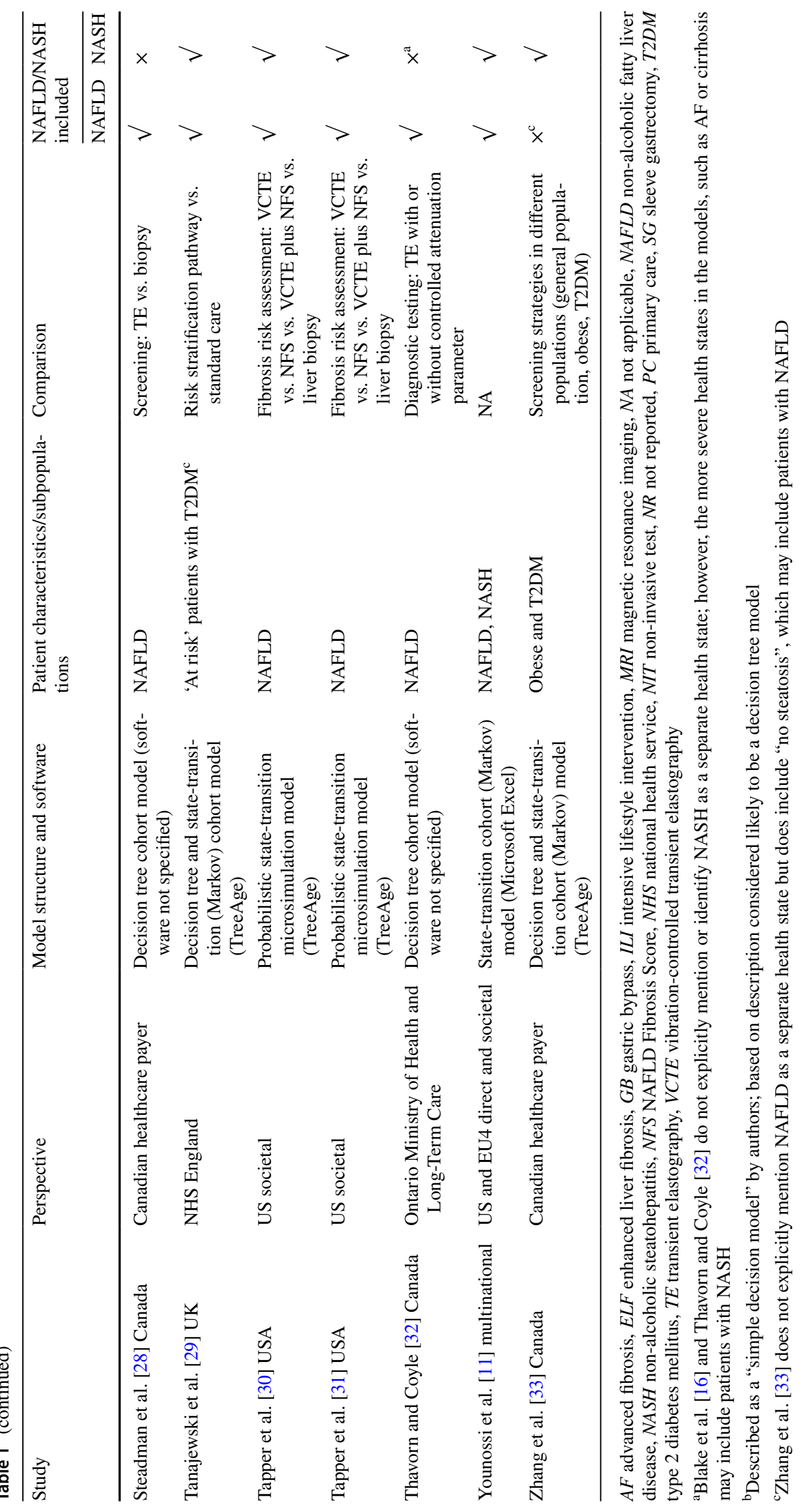


separate entities (Table 1). There was heterogeneity in terms of the way in which NASH was considered, with several using health states specific to NAFLD/NASH. For example, seven articles specifically used 'steatosis' or 'steatohepatitis' health states [11, 17, 18, 21, 30, 31, 33]. In contrast, other investigators used fibrosis states or states such as 'significant liver disease', which corresponded to fibrosis stages (see Table 8 in the ESM for a detailed overview of health states used).

In terms of model structure, most identified models were state-transition models, with or without a preceding decision tree [11, 17, 18, 22-26, 29-31, 33], and four consisted of decision trees alone [16, 21, 28, 32] (one further model was described by the authors as a "simple decision model" and therefore was also considered likely to be a decision tree [19]), all of which used a cohort-based population approach. Three models were described as microsimulation models, thereby using an individual patient-level approach $[25,30$, 31]. As noted, there was substantial heterogeneity between the different models with regard to the consideration of NASH alone, including both NASH and NAFLD but as separate health states or implicitly including NASH but utilising an overarching NAFLD health state. Additionally, of the models that used state-transition elements, two distinct approaches for modelling health states early in the course of the disease were identified (see Table 8 in the ESM). The first and most common approach, used in six publications [17, 18, 22, 25, 26, 29], was to use fibrosis stages. Four studies [17, 25, 26, 29] that used this approach used transition probabilities sourced from a meta-analysis of 11 studies of paired biopsy samples in patients with biopsyproven NAFLD, including 150 patients with NAFL and 261 with NASH [34]. A key limitation of this data source was that, because of low patient numbers for patients with more severe disease, rates of progression were only reported for patients with NAFL or NASH with F0 or F1 disease at baseline (as well as for a pooled population of patients with F0 or F1 disease at baseline). Therefore, it was assumed that the rate of progression for later stages (e.g. F2-F3) was linear. However, one advantage of the use of fibrosis stages is that it allowed for the use of utility and cost data from studies conducted in other liver disease areas. The second commonly used approach for early disease health states was the use of NAFLD- and NASH-specific health states, which was used in three publications $[11,24,33]$. For example, in Younossi et al. [11], early disease health states included "NAFL" and "NASH-non-cirrhotic". Similarly, early disease health states used by Zhang et al. [33] included no steatosis, steatosis, steatohepatitis or fibrosis.

The approach to later stages of disease was relatively consistent for models that used a state-transition approach. Health states typically included cirrhosis, decompensated cirrhosis, HCC and liver transplantation. Notably, three articles (two models) considered HCC in detail [24, 30, 31]. For example, within HCC, Mahady et al. [24] considered states including resection, locoregional therapy, treatment with sorafenib and palliative care. Additionally, three models omitted the possibility of liver transplantation [18, 26, 33]. Although liver transplantation only affects a small proportion of patients, it is associated with high direct medical costs. Transition probabilities for progression from compensated cirrhosis to decompensated cirrhosis or HCC, etc. were frequently sourced from studies in other liver diseases, particularly hepatitis C. Health states associated with comorbid conditions such as diabetes or cardiovascular outcomes were generally not considered in the identified models.

\subsection{Health State Utilities and Costs}

In terms of utility values for different health states, three different approaches were identified. The first approach was to use utilities in patients with other liver diseases, particularly hepatitis $C[17,18,22,23,25,26,33]$. The second approach was to use utility values specific to NAFLD/NASH health states using data from a study by David et al. [35] that assessed QoL in patients with NAFLD/NASH using the 36-item Short Form Survey (SF-36) questionnaire and then mapping these values to the Health Utilities Index (HUI)-2 [11, 33]. The third approach, used by Tanajewski et al. [29], was to use values derived from expert opinion (see Table 9 in the ESM for full details of health state utility values used in identified modelling analyses). Additionally, direct costs associated with late-stage disease/complications such as cirrhosis, decompensated cirrhosis and HCC were also commonly sourced from studies in other liver diseases, again frequently from analyses in patients with hepatitis $\mathrm{C}$ (Table 10 in the ESM). One multinational analysis [11] used a microcosting approach (in addition to previously published costs) for early disease states. Resource use for NAFL and precirrhotic NASH states was determined through consultation with clinical experts, and this was then mapped to national fee schedules [11]. The use of hepatitis $C$ cost data as a proxy was generally attributed to the paucity of cost data specifically relating to NAFLD and NASH. However, as noted by some of the authors of included studies, it is reasonable to assume that the direct costs of the management of states/ complications such as cirrhosis, HCC and liver transplantation will be similar irrespective of the underlying aetiology.

\subsection{Treatment Effects}

Although no drugs are currently indicated for the treatment of NASH, several of the identified articles performed analyses that used data from phase II trials or investigator-initiated studies of drugs used off-label, including 
thiazolidinediones [17, 18, 24, 29, 33], vitamin E [17, 24, $30,31,33$ ] and obeticholic acid [25] (see Table 11 in the ESM for full details). A further eight articles included comparisons using data from studies examining the effects of lifestyle interventions, including weight loss and bariatric surgery [17, 22-24, 26, 30, 31, 33]. Efficacy was typically assessed in small-scale trials in terms of improvement in histology. Typically, a relative risk adjustment to the rate of progression or regression of fibrosis was applied to patients in treatment arms where histologic improvement was reported based on data either directly from trials or-for thiazolidinediones-a metaanalysis. On a related issue, only two articles identified in the review explicitly captured treatment-related adverse events or complications. Specifically, Pearson et al. [25] captured the effects of pruritus and dyslipidaemia associated with the use of obeticholic acid in two phase II trials [36, 37] and Klebanoff et al. [23] captured the risk, QoL detriment and costs associated with minor and major complications associated with bariatric surgery. Klebanoff et al. [23] also included an adjustment for the likelihood of clinical decompensation according to body mass index. The primary and secondary endpoint and safety data available from trials of drugs currently in development will be key determinants of future modelling analyses, but only endpoints that can be used to accurately project long-term outcomes, such as progression of fibrosis, may be suitable for modelling. Drug development guidelines issued by the US FDA and the European Medicines Agency (EMA) advocate that either resolution of steatohepatitis without worsening of fibrosis or improvement in liver fibrosis of at least one stage with no worsening of steatohepatitis be used as primary endpoints in phase III trials of new drugs for NASH [38, 39]. Similarly, a 2019 joint workshop statement from the American Association for the Study of Liver Diseases (AASLD) and European Association for the Study of the Liver (EASL) included resolution of steatohepatitis (with no worsening of fibrosis) or an improvement of at least two points in NAFLD Activity Score (NAS) as recommended primary endpoints in clinical trials [40], but linking such endpoints to long-term outcomes is likely required before they can be used in health economic analyses. The utility of other commonly used secondary endpoints such as change in ALT levels, lobular inflammation or change in hepatic fat levels that cannot be linked with long-term progression may therefore be limited in terms of health economic modelling.

\subsection{Non-Hepatic Outcomes and Consideration of Co-Existing Conditions}

The models identified in the review did not account for changes in metabolic and cardiovascular parameters that are intricately linked with NASH. Several individual studies and meta-analyses have shown that NAFLD is associated with a significantly elevated risk for cardiovascular events [41, 42]; indeed, cardiovascular disease is the leading cause of death in patients with NAFLD [43]. However, cardiovascular endpoints were omitted from the identified models, but if the regression of fibrosis can influence the risk of cardiovascular endpoints, which are frequently associated with high direct medical costs and a substantial decrement in QoL, this may warrant consideration in future health economic models. This is also particularly pertinent for any interventions that target aspects of metabolic pathways, such as lipid metabolism or other parameters such as insulin resistance, which may impact metabolic and cardiovascular risk factors in addition to hepatic outcome measures. Allied to this, co-existing conditions such as obesity and type 2 diabetes mellitus (T2DM) were also not considered as separate health states in the identified models. However, one analysis did perform separate analyses of a NASH screening strategy for a general population, obese patients and patients with T2DM based on different prevalence rates for steatosis and NASH in the three different patient populations [33].

\subsection{Uncertainty and Validation}

The internal and external validation of the identified models was also examined. Only three analyses explicitly reported internal validation processes [17, 29, 32], although deterministic and/or probabilistic sensitivity analyses were performed by most investigators to verify that the model was projecting outcomes as anticipated and determining key drivers of results (Table 2). External validation processes were fully described in three analyses [11, 18, 32]. Where external validation processes were described, these included the comparisons of outcomes with those from previously published studies [18, 32] as well as by comparing those generated using a specialist disease modelling software package (DisMod II) [11].

\subsection{Study Quality}

Assessment of study quality using the checklist by Philips et al. [15] revealed substantial heterogeneity between analyses in terms of quality. Full results of the quality assessment are presented in Table 12 in the ESM. Overall, quality assessment revealed that the identified models were wellsuited to their purpose. For example, using the Philips checklist, all included analyses had "outcomes consistent with the objectives", nearly all (18/19) models were considered to be "appropriate given the decision problem", 16/19 were considered as having "data modelling methodology based on justifiable statistical and epidemiological techniques" and 18/19 analyses were considered as investigating "all feasible and practical options" (see Table 12 in the ESM). 
Table 2 Sensitivity analyses and validation performed in identified modelling studies

\begin{tabular}{|c|c|c|c|c|}
\hline Study & DSA performed & PSA performed & $\begin{array}{l}\text { Internal valida- } \\
\text { tion }\end{array}$ & $\begin{array}{l}\text { External } \\
\text { valida- } \\
\text { tion }\end{array}$ \\
\hline Blake et al. [16] UK & No & Yes & NR & NR \\
\hline Chongmelaxme et al. [17] Thailand & Yes (univariate) & Yes & Yes & No \\
\hline Corey et al. [18] USA & Yes (univariate) & Yes & NR & Yes \\
\hline Crossan et al. [19] UK & Yes (univariate) & Yes & NR & NR \\
\hline Crossan et al. [20] UK & Yes (univariate) & No & NR & NR \\
\hline Eddowes et al. [21] UK & No & No & NR & NR \\
\hline Klebanoff et al. [22] USA & Yes & Yes & NR & NR \\
\hline Klebanoff et al. [23] USA & Yes (univariate) & Yes & NR & NR \\
\hline Mahady et al. [24] Australia & Yes (univariate and multivariate) & No & NR & NR \\
\hline Pearson et al. [25] USA & Yes (univariate) & No & NR & NR \\
\hline Phisalprapa et al. [26] Thailand & Yes (univariate) & Yes & NR & NR \\
\hline Srivastava et al. [27] UK & Yes (univariate) & No & NR & NR \\
\hline Steadman et al. [28] Canada & Yes (univariate) & Yes & NR & NR \\
\hline Tanajewski et al. [29] UK & Yes (univariate and multivariate) & Yes & Yes & NR \\
\hline Tapper et al. [30] USA & Yes (univariate) & Yes & NR & NR \\
\hline Tapper et al. [31] USA & Yes (univariate) & Yes & NR & NR \\
\hline Thavorn and Coyle [32] Canada & Yes (univariate) & Yes & Yes & Yes \\
\hline Younossi et al. [11] multinational & Yes (univariate) & No & NR & Yes \\
\hline Zhang et al. [33] Canada & Yes (univariate and multivariate) & No & NR & NR \\
\hline
\end{tabular}

$D S A$ deterministic sensitivity analyses, $N R$ not reported, $P S A$ probabilistic sensitivity analyses

\section{Discussion}

\subsection{Overview of Existing Models}

Overall, review of published health economic models of NAFLD/NASH demonstrated that most economic models of NAFLD used a state-transition model approach and that the approach to modelling late-stage complications was generally consistent, although different approaches were used to model earlier disease and the quality of existing models was heterogenous. Additionally, most of the published costeffectiveness analyses in NAFLD/NASH were conducted for North American or European settings, with only three publications from another country (Thailand [17, 26] and Australia [24]). However, the literature searches were limited to studies published in English, so it is feasible that other studies in non-English languages may be available but not included here. The literature review also highlighted a general paucity of input data for economic analyses in this area (Table 3).

In particular, health state utilities, complication costs and transition probabilities were frequently sourced from studies conducted in other liver disease areas, particularly hepatitis C. Whereas the use of input data from other disease areas may be suboptimal, it should be noted that model developers are restricted in terms of the available data, and-where data gaps exist - are faced with having to evaluate the relative merits and limitations of different alternatives or proxy sources. For example, health technology assessment (HTA) agencies in some countries, such as Sweden, prefer analyses that use health state utilities elicited using direct methods such as standard gamble or time trade-off, and others

Table 3 Existing data gaps for NAFLD/NASH models

NAFLD/NASH-specific health state utility values, particularly consideration of QoL in patients with NASH with comorbid conditions such as obesity or T2DM

NAFLD/NASH-specific costs for health states

NAFLD/NASH-specific transition probabilities or risk equations for disease progression beyond F1 stage

Data linking surrogate endpoints to hard clinical outcomes, for example, through the development of risk equations

Data that may allow for the identification of fast/slow progressors and predictors of rate of progression

$N A F L D$ non-alcoholic fatty liver disease, $N A S H$ non-alcoholic steatohepatitis, $Q o L$ quality of life, $T 2 D M$ type 2 diabetes mellitus 
prefer indirect methods, with HTAs in countries such as the UK, Netherlands and Belgium specifically mentioning the EuroQoL 5-Dimensions (EQ-5D) tool [44]. However, utility values for NAFLD-/NASH-specific health states and specifically utility values elicited using the EQ-5D are lacking. This leaves model developers in countries such as the UK with the alternatives of using NAFLD/NASH-specific values elicited using the SF-36 and mapped to the HUI-2 or using EQ-5D values for different fibrosis stages elicited from patients with other liver diseases (primarily hepatitis $\mathrm{B}$ or C). However, this issue is likely limited to early disease states as utility values (and also direct costs) for late-stage complications, such as decompensated cirrhosis, are unlikely to be significantly influenced by the underlying aetiology of liver disease.

Model developers are also faced with the issue of how best to capture the QoL decrements associated with health states that frequently co-exist in patients with NAFLD/ NASH, such as obesity and T2DM while avoiding 'double counting' of QoL decrements. One alternative is to use a multiplicative approach to model the QoL decrement in instances of comorbid conditions, which is accepted by some HTA agencies, including the UK National Institute for Health and Care Excellence (NICE), but this approach also relies on assumptions. Three analyses identified in the review assigned a mean utility value of 1.0 to the states of NAFLD and simple steatosis [30, 31, 33], which—given the mean age of NAFLD/NASH populations and high prevalence of obesity and T2DM within this patient populationmay be unrealistic. Current limitations around the lack of data could potentially be overcome with data from clinical trials of novel drugs, particularly if trials include patientreported outcomes such as QoL. Further studies specifically in patients with NASH are needed to address some data gaps, such as QoL and costs and linking surrogate outcomes to hard clinical endpoints. These data are likely to become available in the future with the ongoing development of new drugs for the treatment of NASH.

\subsection{Considerations for Future Model Development}

A key consideration for future model developers is how best to capture the progression and regression of liver disease. The approach used by several investigators to date was to model progression through stages of fibrosis using transition probabilities derived from a meta-analysis by Singh et al. [34]. However, this approach is associated with a number of limitations. First, Singh et al. [34] only reported mean rates of progression for patients with stage 0 or 1 fibrosis at baseline for patients with NAFLD, NAFL and NASH. Consequently, in the absence of data, model developers necessarily assumed that the rate of progression between different fibrosis stages was linear. In hepatitis $\mathrm{C}$, fibrosis progression estimates are frequently sourced from Thein et al. [45], in which the rate of progression between different fibrosis stages was similar. However, whether this assumption is transferable across different liver disease areas has yet to be elucidated. Additionally, in their meta-analysis Singh et al. [34] noted that the rate of progression was heterogenous and that $21 \%$ of patients with NAFLD could be classified as "rapid progressors" and progressed from stage 0 to stage 3 or 4 fibrosis over a mean \pm standard deviation of $5.9 \pm 3.7$ years. However, Singh et al. [34] did not present separate progression rates for rapid versus slow progressors. Factors including older age, presence of T2DM, higher steatosis grade and low aspartate transaminase (AST):alanine transaminase (ALT) ratio have previously been linked with more rapid progression; [34] however, differential rates of progression for rapid versus slow progressors have not been captured in the identified models. As such, this is an area that may warrant further investigation and potentially the development of risk equations linking patient-related factors such as age or presence of comorbidities as well as surrogate endpoints such as steatosis or AST:ALT ratio to rate of progression. The ability to model progression and identify and perform subgroup analyses in rapid progressors would be a useful feature worthy of consideration for inclusion in future models. Another aspect that was largely overlooked in identified models was the intricate association between NAFLD/NASH and obesity, T2DM and cardiovascular outcomes as well as the possibility for regression of disease and the downstream implications of this, such as the effect on cardiovascular risk factors or even cardiovascular outcomes $[46,47]$. One group partially addressed this issue by performing separate analyses in patients who were obese or had T2DM at baseline [33], but this approach does not fully capture the long-term complication risks associated with frequently co-existing conditions such as T2DM.

The focus on changes in fibrosis stage alone in both clinical studies and modelling analysis means that potentially clinically meaningful changes in other histologic parameters such as steatosis, ballooning, lobular inflammation and portal inflammation, as well as changes in cardiometabolic risk factors, may not be captured. Moreover, in addition to changes in fibrosis, recommended primary endpoints for phase III trials in patients with pre-cirrhotic NASH include resolution of NASH without worsening of fibrosis or $\geq 1$ stage reduction in fibrosis (without worsening of steatosis in EMA and FDA guidance), or $\geq 2$-point reduction in NAS with $\geq 1$-point reduction in either lobular inflammation or hepatocellular ballooning and no worsening of fibrosis [38, 39, 48]. In AASLD/EASL guidance, recommended secondary endpoints in phase II/III clinical trials include cardiometabolic risk factors [48]. However, for endpoints to be suitable for economic modelling, they must be definitively linked with long-term outcomes such as occurrence 
of cirrhosis, HCC or liver-related mortality. Long-term data linking histologic outcomes such as improvement in NAS to long-term outcomes are scarce, which presents a barrier to their use in modelling analyses. Indeed, evidence to date suggests that fibrosis is the only endpoint that can currently be independently linked with long-term outcomes, including liver-related morbidity and mortality $[49,50]$.

With regard to the structure of future models for NASH, given the heterogeneity of NASH as a condition, it is feasible that a microsimulation approach rather than a cohort-level approach may represent the optimal approach for future models. In particular, the microsimulation approach (unlike the cohort approach) allows for heterogeneity to be captured on an individual patient level. It also offers a flexible approach and allows for the modelling of factors that have non-linear relationships with model outcomes, and NASH has been shown to have non-linear disease pathology (exemplified by the existence of fast progressors and slow progressors) [51].

\subsection{The Future of Treatment for Non-Alcoholic Fatty Liver Disease/Non-Alcoholic Steatohepatitis}

There is currently an unmet clinical need for efficacious pharmacological treatments for NASH. Lifestyle modifications such as weight loss have been associated with regression of NASH, but some patients find lifestyle modifications difficult to sustain [52]. Indeed, European guidelines state that bariatric surgery can be considered for obese patients who have been unresponsive to lifestyle modifications and pharmacotherapy [12]. Bariatric surgery has been shown to reduce liver fat levels via improvements in obesity and diabetes but is also associated with a risk of peri-operative and post-operative complications [12]. Additionally, the use of pioglitazone and vitamin E is recommended in both European and US guidelines [1, 12]; however, both agents are used off label, and no drugs are currently indicated for use in NASH in either Europe or the USA. This has implications in terms of the availability of robust clinical data for comparator treatments and for demonstrating cost effectiveness relative to the current standard of care (SoC). In the absence of approved drug treatments, the current $\mathrm{SoC}$ for patients with NASH is lifestyle modification and weight loss. This means that in future trials of novel drugs, the comparison may be active treatment plus lifestyle modification versus placebo plus lifestyle modification, which may necessitate the use of indirect comparisons to compare different novel drug treatments. Allied to this, several of the agents currently in development for the treatment of NASH target processes such as oxidative stress or insulin resistance and therefore may be associated with therapeutic benefits extending beyond the hepatocyte, which should also be taken into account in health economic modelling analyses.

\subsection{Transparency in Future Economic Models}

Future economic models of NAFLD/NASH should conform to good practice standards. These include but are not limited to appropriately and adequately representing the disease process, balancing the necessary complexity required to appropriately capture all necessary aspects of the disease process but not being so complex as to be unclear and compromise transparency, as well as appropriately considering uncertainty, using conservative assumptions in the absence of data and having appropriate validation and sufficient transparency to enable others to reproduce the model [53-55]. In particular, transparency and validation are regarded as crucial for imbuing the model with credibility for interested stakeholders [56]. This review of identified NAFLD/NASH models revealed substantial heterogeneity in terms of the reporting of models and also a general lack of reporting of validation processes.

Additionally, a consistent limitation identified across all included publications was that structural uncertainty was not addressed. Other commonly identified limitations included failure to assess the quality of input data (identified in 13 publications), failure to provide evidence that the mathematical logic of the model had been thoroughly tested before use (13 publications) and failure to present evidence of comparison or cross-validation with other models (nine publications). However, it should be noted that an inherent limitation of quality assessment is that findings may be negatively influenced in instances where the quality of reporting of the model structure, development and input data sources is poor or restricted because of stringent word limits applied by some journals, which may mean that the quality of the reporting of an analysis may not accurately reflect the quality of the actual modelling analysis per se. However, to circumvent this, authors could select journals that allow publication of supplementary online material for interested readers.

A lack of transparency relating to some aspects of health economic models in other liver disease areas, such as chronic hepatitis B, has also been reported [57]. One approach to enhancing transparency and validation is the formation of informal networks within disease areas to actively promote this. For example, the Mount Hood Diabetes Challenge Network is an informal association of diabetes modellers that holds regular meetings with an aim of promoting transparency though validation and allows for internal, external and cross-validation of available diabetes models [56]. Previous meetings have involved transparency challenges wherein model developers are tasked with reproducing the results of clinical and cost-effectiveness analyses, with differences between models compared and rationale sought for any major differences $[56,58]$. The Mount Hood group have also developed the Diabetes Modelling Input Checklist, a 
disease-specific checklist [58]. Corresponding networks in other disease areas could similarly promote transparency and validation. Another approach would be more widespread use of validation checklists such as the AdViSHE tool published by Vemer et al. [59] or the establishment of other diseasespecific modelling checklists, which may be more useful in terms of capturing nuances specific to individual therapy areas. None of the articles identified in this review used the AdViSHE tool for validation, although it should be noted that 6 of the 19 included articles predated the publication of the AdViSHE tool. Further, with regard to validation processes specifically, peer reviewers have an integral role to play by requesting that model developers include details of validation processes in publications. Additionally, another potential caveat of economic models created de novo is that they are often designed to be highly product specific and, while this has the advantage of accurately capturing aspects such as treatment-related adverse events, non-product-specific models have the advantage of enabling comparison of findings across different analyses and treatments.

\section{Conclusions}

Available models were associated with limitations, largely driven by a lack of data specific to NAFLD/NASH patient populations; however, a key limitation with regard to model structure was the consistent omission of health states relating to conditions that frequently exist alongside NASH, such as obesity and T2DM, as well as the omission of the increased risk for cardiovascular disease reported in patients with NASH. High-quality, transparent, validated health economic models of NASH will be required to evaluate the cost effectiveness of novel treatments currently in development, particularly for compounds that may target both hepatic and non-hepatic outcomes.

\begin{abstract}
Acknowledgements The authors thank Giorgia Magnatti, Tim Morton and Leticia Barcena of DRG Abacus for assistance on further searches and data extraction. The authors also thank Jayne Smith-Palmer and Barnaby Hunt of Ossian Health Economics and Communications for medical writing assistance and Laura Escalante Santos of Oxford PharmaGenesis for editorial assistance.
\end{abstract}

Author contributions Pierre Johansen provided the study concept. Pierre Johansen, Daniel Howard and Ryan Bishop provided the study design. Daniel Howard and Ryan Bishop performed the data extraction. All authors contributed to the analysis and interpretation of the data and contributed to the drafting of the paper, critically revised the paper for intellectual content, were involved in the final approval of the version to be published and agreed to be accountable for all aspects of the work. Pierre Johansen is the overall guarantor.

Data availability Data sharing not applicable to this article as no new datasets were generated or analysed during the current study.

\section{Compliance with Ethical Standards}

Funding Funding for this review was provided by Novo Nordisk A/S.

Disclosures Daniel Howard and Ryan Bishop are former or current employees of DRG Abacus, which has received consulting fees from Novo Nordisk A/S. Pierre Johansen, Søren Ilsøe Moreno and Kristine Buchholtz are current employees of Novo Nordisk A/S. Pierre Johansen is also a shareholder of Novo Nordisk A/S.

Open Access This article is licensed under a Creative Commons Attribution-NonCommercial 4.0 International License, which permits any non-commercial use, sharing, adaptation, distribution and reproduction in any medium or format, as long as you give appropriate credit to the original author(s) and the source, provide a link to the Creative Commons licence, and indicate if changes were made. The images or other third party material in this article are included in the article's Creative Commons licence, unless indicated otherwise in a credit line to the material. If material is not included in the article's Creative Commons licence and your intended use is not permitted by statutory regulation or exceeds the permitted use, you will need to obtain permission directly from the copyright holder.To view a copy of this licence, visit http://creativecommons.org/licenses/by-nc/4.0/.

\section{References}

1. Chalasani N, Younossi Z, Lavine JE, Charlton M, Cusi K, Rinella $\mathrm{M}$, et al. The diagnosis and management of nonalcoholic fatty liver disease: practice guidance from the American Association for the Study of Liver Diseases. Hepatology. 2018;67:328-57.

2. Neuschwander-Tetri BA. Non-alcoholic fatty liver disease. BMC Med. 2017;15:45.

3. Younossi ZM, Koenig AB, Abdelatif D, Fazel Y, Henry L, Wymer M. Global epidemiology of nonalcoholic fatty liver disease-metaanalytic assessment of prevalence, incidence, and outcomes. Hepatology. 2016;64:73-84.

4. Machado MV, Diehl AM. Pathogenesis of nonalcoholic steatohepatitis. Gastroenterology. 2016;150:1769-77.

5. Schuppan D, Surabattula R, Wang XY. Determinants of fibrosis progression and regression in NASH. J Hepatol. 2018;68:238-50.

6. Hossain N, Afendy A, Stepanova M, Nader F, Srishord M, Rafiq N, et al. Independent predictors of fibrosis in patients with nonalcoholic fatty liver disease. Clin Gastroenterol Hepatol. 2009; 7:1224-9.

7. Kim D, Touros A, Kim WR. Nonalcoholic fatty liver disease and metabolic syndrome. Clin Liver Dis. 2018;22:133-40.

8. Khashab MA, Liangpunsakul S, Chalasani N. Nonalcoholic fatty liver disease as a component of the metabolic syndrome. Curr Gastroenterol Rep. 2008;10:73-80.

9. Dyson JK, Anstee QM, McPherson S. Non-alcoholic fatty liver disease: a practical approach to diagnosis and staging. Frontline Gastroenterol. 2014;5:211-8.

10. Spengler EK, Loomba R. Recommendations for diagnosis, referral for liver biopsy, and treatment of nonalcoholic fatty liver disease and nonalcoholic steatohepatitis. Mayo Clin Proc. 2015;90:1233-46.

11. Younossi ZM, Blissett D, Blissett R, Henry L, Stepanova M, Younossi $\mathrm{Y}$, et al. The economic and clinical burden of nonalcoholic fatty liver disease in the United States and Europe. Hepatology. 2016;64:1577-86. 
12. European Association for the Study of the Liver (EASL); European Association for the Study of Diabetes (EASD); European Association for the Study of Obesity (EASO). EASL-EASDEASO Clinical Practice Guidelines for the management of nonalcoholic fatty liver disease. J Hepatol. 2016;64:1388-402.

13. Clinicaltrials.gov. Active phase II/III clinical trials in nonalcoholic steatohepatitis. https://clinicaltrials.gov/ct2/resul ts $?$ cond $=$ Non-alcoholic + Steat ohepatitis \& recrs $=d \&$ age $\mathrm{v}=\&$ gndr $=\&$ type $=\& \mathrm{rslt}=\&$ phase $=1 \&$ phase $=2 \&$ Search $=$ Apply . Accessed 17 Dec 2019.

14. Higgins JPT, Green S. Cochrane handbook for systematic reviews of interventions version 5.1.0. https://training.cochrane.org/handb ook. Accessed 31 Jul 2019.

15. Philips Z, Ginnelly L, Sculpher M, Claxton K, Golder S, Riemsma $\mathrm{R}$, et al. Review of guidelines for good practice in decision-analytic modelling in health technology assessment. Health Technol Assess. 2004;8(36):1-158.

16. Blake L, Duarte RV, Cummins C. Decision analytic model of the diagnostic pathways for patients with suspected non-alcoholic fatty liver disease using non-invasive transient elastography and multiparametric magnetic resonance imaging. BMJ Open. 2016;6(9):e010507.

17. Chongmelaxme B, Phisalprapa P, Sawangjit R, Dilokthornsakul $\mathrm{P}$, Chaiyakunapruk N. Weight reduction and pioglitazone are costeffective for the treatment of non-alcoholic fatty liver disease in Thailand. Pharmacoeconomics. 2019;37:267-78.

18. Corey KE, Klebanoff MJ, Tramontano AC, Chung RT, Hur C. Screening for nonalcoholic steatohepatitis in individuals with type 2 diabetes: a cost-effectiveness analysis. Dig Dis Sci. 2016;61:2108-17.

19. Crossan C, Tsochatzis EA, Longworth L, Gurusamy K, Davidson $\mathrm{B}$, Rodríguez-Perálvarez M, et al. Cost-effectiveness of non-invasive methods for assessment and monitoring of liver fibrosis and cirrhosis in patients with chronic liver disease: systematic review and economic evaluation. Health Technol Assess. 2015;19:1-409.

20. Crossan C, Majumdar A, Srivastava A, Thorburn D, Rosenberg W, Pinzani M, et al. Referral pathways for patients with NAFLD based on non-invasive fibrosis tests: diagnostic accuracy and cost analysis. Liver Int. 2019;39:2052-60.

21. Eddowes PJ, McDonald N, Davies N, Semple SIK, Kendall TJ, Hodson J, et al. Utility and cost evaluation of multiparametric magnetic resonance imaging for the assessment of non-alcoholic fatty liver disease. Aliment Pharmacol Ther. 2018;47:631-44.

22. Klebanoff MJ, Corey KE, Chhatwal J, Kaplan LM, Chung RT, Hur C. Bariatric surgery for nonalcoholic steatohepatitis: a clinical and cost-effectiveness analysis. Hepatology. 2017;65:1156-64.

23. Klebanoff MJ, Corey KE, Samur S, Choi JG, Kaplan LM, Chhatwal J, et al. Cost-effectiveness analysis of bariatric surgery for patients with nonalcoholic steatohepatitis cirrhosis. JAMA Netw Open. 2019;2:e190047.

24. Mahady SE, Wong G, Craig JC, George J. Pioglitazone and vitamin $\mathrm{E}$ for nonalcoholic steatohepatitis: a cost utility analysis. Hepatology. 2012;56:2172-9.

25. Pearson SD, Banken R, Chapman RH, Russo ET, Loos AM, Emond SK et al. Obeticholic acid for the treatment of nonalcoholic steatohepatitis: comparative clinical effectiveness and value. Institute for Clinical and Economic Review (ICER); 2016. https ://icer-review.org/wp-content/uploads/2016/06/NECEPAC_OCA_ NASH_Evidence_Report_062316.pdf. Accessed 31 Jul 2019.

26. Phisalprapa P, Supakankunti S, Charatcharoenwitthaya P, Apisarnthanarak P, Charoensak A, Washirasaksiri C, et al. Cost-effectiveness analysis of ultrasonography screening for nonalcoholic fatty liver disease in metabolic syndrome patients. Medicine (Baltimore). 2017;96:e6585.

27. Srivastava A, Jong S, Gola A, Gailer R, Morgan S, Sennett K. Cost-comparison analysis of FIB-4, ELF and fibroscan in community pathways for non-alcoholic fatty liver disease. BMC Gastroenterol. 2019;19(1):122.

28. Steadman R, Myers RP, Leggett L, Lorenzetti D, Noseworthy T, Rose $\mathrm{S}$, et al. A health technology assessment of transient elastography in adult liver disease. Can J Gastroenterol. 2013;27:149-58.

29. Tanajewski L, Harris R, Harman DJ, Aithal GP, Card TR, Gkountouras G, Berdunov V, Guha IN, Elliott RA. Economic evaluation of a community-based diagnostic pathway to stratify adults for non-alcoholic fatty liver disease: a Markov model informed by a feasibility study. BMJ Open. 2017;7(6):e015659.

30. Tapper EB, Sengupta N, Hunink MG, Afdhal NH, Lai M. Costeffective evaluation of nonalcoholic fatty liver disease with NAFLD fibrosis score and vibration controlled transient elastography. Am J Gastroenterol. 2015;110:1298-304 (erratum in Am J Gastroenterol. 2016;111(3):446).

31. Tapper EB, Hunink MG, Afdhal NH, Lai M, Sengupta N. Costeffectiveness analysis: risk stratification of nonalcoholic fatty liver disease (NAFLD) by the primary care physician using the NAFLD fibrosis score. PLoS One. 2016;11(2):e0147237.

32. Thavorn K, Coyle D. Transient elastography and controlled attenuation parameter for diagnosing liver fibrosis and steatosis in Ontario: an economic analysis. Ont Health Technol Assess Ser. 2015;15:1-58.

33. Zhang E, Wartelle-Bladou C, Lepanto L, Lachaine J, Cloutier G, Tang A. Cost-utility analysis of nonalcoholic steatohepatitis screening. Eur Radiol. 2015;25:3282-94.

34. Singh S, Allen AM, Wang Z, Prokop LJ, Murad MH, Loomba R. Fibrosis progression in nonalcoholic fatty liver vs. nonalcoholic steatohepatitis: a systematic review and meta-analysis of pairedbiopsy studies. Clin Gastroenterol Hepatol. 2015;13(4):643-54 e1-9.

35. David K, Kowdley KV, Unalp A, Kanwal F, Brunt EM, Schwimmer JB, NASH CRN Research Group. Quality of life in adults with nonalcoholic fatty liver disease: baseline data from the nonalcoholic steatohepatitis clinical research network. Hepatology. 2009;49:1904-12.

36. Neuschwander-Tetri BA, Loomba R, Sanyal AJ, Lavine JE, Van Natta ML, Abdelmalek MF, Chalasani N, Dasarathy S, Diehl AM, Hameed B, Kowdley KV, McCullough A, Terrault N, Clark JM, Tonascia J, Brunt EM, Kleiner DE, Doo E, NASH Clinical Research Network. Farnesoid X nuclear receptor ligand obeticholic acid for non-cirrhotic, non-alcoholic steatohepatitis (FLINT): a multicentre, randomised, placebo-controlled trial. Lancet. 2015;385(9972):956-65.

37. Mudaliar S, Henry RR, Sanyal AJ, Morrow L, Marschall HU, Kipnes M, Adorini L, Sciacca CI, Clopton P, Castelloe E, Dillon P, Pruzanski M, Shapiro D. Efficacy and safety of the farnesoid $\mathrm{X}$ receptor agonist obeticholic acid in patients with type 2 diabetes and nonalcoholic fatty liver disease. Gastroenterology. 2013;145(3):574-574.e1.

38. Food and Drug Administration. 2018. Noncirrhotic non-alcoholic steatohepatitis with liver fibrosis: developing drugs for treatment: guidance for industry. https://www.fda.gov/media /119044/download. Accessed 19 Sep 2019.

39. European Medicines Agency. 2018. Reflection paper on regulatory requirements for the development of medicinal products for chronic non-infectious liver diseases (PBC, PSC, NASH). https://www.ema.europa.eu/en/documents/scientific-guideline/ reflection-paper-regulatory-requirements-development-medic inal-products-chronic-non-infectious-liver_en.pdf. Accessed 19 Sep 2019.

40. Sanyal AJ, Brunt EM, Kleiner DE, Kowdley KV, Chalasani N, Lavine JE, et al. Endpoints and clinical trial design for nonalcoholic steatohepatitis. Hepatology. 2011;54:344-53.

41. Mahfood Haddad T, Hamdeh S, Kanmanthareddy A, Alla VM. Nonalcoholic fatty liver disease and the risk of clinical 
cardiovascular events: a systematic review and meta-analysis. Diabetes Metab Syndr. 2017;11(Suppl 1):S209-16.

42. Targher G, Byrne CD, Lonardo A, Zoppini G, Barbui C. Nonalcoholic fatty liver disease and risk of incident cardiovascular disease: a meta-analysis. J Hepatol. 2016;65:589-600.

43. Adams LA, Anstee QM, Tilg H, Targher G. Non-alcoholic fatty liver disease and its relationship with cardiovascular disease and other extrahepatic diseases. Gut. 2017;66:1138-53.

44. Eunethta. Methods for health economic evaluations - a guideline based on current practices in Europe. 2015. https://www.eunet hta.eu/wp-content/uploads/2018/03/Methods_for_health_econo mic_evaluations.pdf. Accessed 14 Aug 2019.

45. Thein HH, Yi Q, Dore GJ, Krahn MD. Estimation of stage-specific fibrosis progression rates in chronic hepatitis $\mathrm{C}$ virus infection: a meta-analysis and meta-regression. Hepatology. 2008;48:418-31.

46. Corey KE, Wilson LA, Altinbas A, Yates KP, Kleiner DE, Chung RT, NASH Clinical Research Network, et al. Relationship between resolution of non-alcoholic steatohepatitis and changes in lipoprotein sub-fractions: a post-hoc analysis of the PIVENS trial. Aliment Pharmacol Ther. 2019;49:1205-13.

47. Corey KE, Vuppalanchi R, Wilson LA, Cummings OW, Chalasani $\mathrm{N}$, NASH CRN. NASH resolution is associated with improvements in HDL and triglyceride levels but not improvement in LDL or non-HDL-C levels. Aliment Pharmacol Ther. 2015;41:301-9.

48. Rinella ME, Tacke F, Sanyal AJ, Anstee QM; Participants of the AASLD/EASL Workshop. Report on the AASLD/EASL joint workshop on clinical trial endpoints in NAFLD. J Hepatol. 2019. https://doi.org/10.1016/j.jhep.2019.04.019 (Epub ahead of print).

49. Angulo P, Kleiner DE, Dam-Larsen S, Adams LA, Bjornsson ES, Charatcharoenwitthaya $\mathrm{P}$, et al. Liver fibrosis, but no other histologic features, is associated with long-term outcomes of patients with nonalcoholic fatty liver disease. Gastroenterology. 2015;149(389-97):e10.

50. Younossi ZM, Loomba R, Rinella ME, Bugianesi E, Marchesini G, Neuschwander-Tetri BA, et al. Current and future therapeutic regimens for nonalcoholic fatty liver disease and nonalcoholic steatohepatitis. Hepatology. 2018;68:361-71.

51. Davis S, Stevenson M, Tappenden P, Wailoo AJ. NICE DSU Technical Support Document 15: Cost-effectiveness modelling using patient-level simulation. 2014. http://www.nicedsu.org.uk.
52. Vilar-Gomez E, Martinez-Perez Y, Calzadilla-Bertot L, TorresGonzalez A, Gra-Oramas B, Gonzalez-Fabian L, et al. Weight loss through lifestyle modification significantly reduces features of nonalcoholic steatohepatitis. Gastroenterology. 2015;149:367-78.

53. Caro JJ, Briggs AH, Siebert U, Kuntz KM, ISPOR-SMDM Modeling Good Research Practices Task Force. Modeling good research practices-overview: a report of the ISPOR-SMDM Modeling Good Research Practices Task Force-1. Value Health. 2012;15:796-803.

54. Roberts M, Russell LB, Paltiel AD, Chambers M, McEwan P, Krahn M, ISPOR-SMDM Modeling Good Research Practices Task Force. Conceptualizing a model: a report of the ISPORSMDM Modeling Good Research Practices Task Force-2. Value Health. 2012;15:804-11.

55. Eddy DM, Hollingworth W, Caro JJ, Tsevat J, McDonald KM, Wong JB, ISPOR-SMDM Modeling Good Research Practices Task Force. Model transparency and validation: a report of the ISPOR-SMDM Modeling Good Research Practices Task Force-7. Value Health. 2012;15:843-50.

56. Kent S, Becker F, Feenstra T, Tran-Duy A, Schlackow I, Tew $\mathrm{M}$, et al. The challenge of transparency and validation in health economic decision modelling: a view from Mount Hood. Pharmacoeconomics. 2019. https://doi.org/10.1007/s40273-019-00825-1 (Epub ahead of print).

57. Wigfield P, Sbarigia U, Hashim M, Vincken T, Heeg B. Are published health economic models for chronic hepatitis B appropriately capturing the benefits of $\mathrm{HBs} A g$ loss? A systematic literature review. Pharmacoecon Open. 2019. https://doi.org/10.1007/s4166 9-019-00175-w (Epub ahead of print).

58. Palmer AJ, Si L, Tew M, Hua X, Willis MS, Asseburg C, et al. Computer modeling of diabetes and its transparency: a report on the eighth Mount Hood challenge. Value Health. 2018;21:724-31.

59. Vemer P, Corro Ramos I, van Voorn GA, Al MJ, Feenstra TL. AdViSHE: a validation-assessment tool of health-economic models for decision makers and model users. Pharmacoeconomics. 2016;34:349-61.

\section{Affiliations}

\section{Pierre Johansen $^{1}(1) \cdot$ Daniel Howard ${ }^{2} \cdot$ Ryan Bishop $^{3} \cdot$ Søren Ilsøe Moreno ${ }^{1} \cdot$ Kristine Buchholtz $^{1}$}

Pierre Johansen

pirj@novonordisk.com

1 Novo Nordisk A/S, Søborg, Denmark

2 DRG Abacus, Manchester, UK
3 DRG Abacus, Bicester, UK 